\title{
A Low Complexity 2D Pattern Synthesis Algorithm for Cylindrical Array
}

\author{
Chao Liu, Zhizhong Ding, and Xiaoping Liu \\ Department of Communication Engineering, Hefei University of Technology, Hefei, Anhui 230009, China \\ Correspondence should be addressed to Chao Liu; disneyl@hfut.edu.cn
}

Received 12 August 2013; Accepted 11 October 2013

Academic Editor: Atsushi Mase

Copyright (C) 2013 Chao Liu et al. This is an open access article distributed under the Creative Commons Attribution License, which permits unrestricted use, distribution, and reproduction in any medium, provided the original work is properly cited.

\begin{abstract}
This paper proposes a 2D pattern synthesis algorithm for cylindrical array. According to the geometric characteristic of cylinder, we can regard a cylindrical array as an equivalent linear array whose elements are identical circular subarrays. Therefore, the beam pattern can be obtained by the product of the array factor of linear array and beam pattern of circular subarray. Then, the $2 \mathrm{D}$ beamforming can be realized by two $1 \mathrm{D}$ beamforming processes. We can prove that the complex excitation vector of a cylindrical array is the Kronecker product of linear array's weight vector and circular array's weight vector. By this algorithm of decomposition and reconstruction, the computational complexity of $2 \mathrm{D}$ beamforming could be significantly reduced. Finally, simulation results further illustrate the validity of the proposed method.
\end{abstract}

\section{Introduction}

Conformal arrays can be commodiously mounted on a curved surface of platforms, such as aerial vehicles and fighter aircraft, and provide aerodynamic shape compatible with the corresponding fuselage. This conformal design leads to an excellent aerodynamic performance and thus has an extremely wide range of applications due to its advantages of low radar cross section (RCS), large surveillance coverage, and volume saving [1]. Although conformal arrays are widely used in many areas, the pattern synthesis of such arrays is still a challenge because of the complexity induced by the unusual configuration. For the arrays, the simple characteristics associated with linear or planar arrays do not hold, and most of the conventional methods would not work.

In the recent years, pattern synthesis of conformal arrays has attracted increasing attention and a wide variety of techniques have been developed for the pattern synthesis of conformal arrays. In [2], Bucci et al. propose a method to solve the pattern synthesis problem of arbitrary planar array. They alternately found the projection on the upper and lower bounds of the desired pattern to control the shape of main lobe and the side lobe level (SLL). Afterwards, the method was extended to conformal arrays. Considering the embedded element radiation pattern, Steyskal used it in a conformal wing array [3]. Tseng and Griffiths provide a new iterative method in [4] to achieve the desired beam pattern of arbitrary arrays by controlling the side lobe peaks. But the number of side lobe peaks that it can control is limited. In [5], Vaskelainen presents an iterative leastsquares method by assigning different weight values to different directions. He further presents a modified leastsquares optimization method with linear constraints in [6] to obtain a prescribed shape of main lobe. In [7], Olen and Compton propose another method based on adaptive array theory. In this method, they first set excessive artificial interference signals in observation area outside the main lobe and then adaptively adjust the intensities of these interference signals to control the side lobe level. In [8], Zhou and Ingram improve the method above. They only allow iterations occurred in the main lobe and among side lobe peaks. The method can provide more convenient main lobe shape control, but finding the location of side lobe peaks might increase computational complexity. In [9], Guo et al. use linearly constrained minimum variance (LCMV) criterion to achieve the desired beam pattern with a flat top main lobe. Dohmen et al. give a synthesis method of conformal array in [10] to design both the copolarized and cross-polarized 
patterns. In [11], Zou et al. present an adaptive beamforming method with low level of polarization components based on geometric algebra. The synthesis problem of an arbitrary array antenna can be seen as a general optimization problem. Therefore, intelligent optimization algorithms can also be used to solve this problem. Genetic algorithms (GA) are applied to complete this kind of optimization in [12, 13]. Simulated annealing and particle swarm optimization (PSO) also have good performance of global optimization and are used in the area of pattern synthesis $[14,15]$.

Most of these methods are applied to general conformal arrays and need an iterative process. The computational complexity is acceptable in $1 \mathrm{D}$ condition. However, the amount of calculation will significantly increase when they are applied to $2 \mathrm{D}$ beamforming. Some researchers have realized this problem and began to find fast algorithms [16]. Nevertheless, the structure information is not taken into consideration. Most of the conformal arrays have regular structures which may be helpful to the array beamforming. In [17], the authors indicate that the cylindrical array can be treated as a linear array while beamforming. However, they did not touch upon the $2 \mathrm{D}$ beamforming problem.

In this paper, we present a new method to solve the 2D beam pattern synthesis for cylindrical arrays. According to the geometry feature of cylinder, we treat the cylindrical array as an equivalent linear array whose elements are identical circular subarrays. Since the linear array and circular array are orthogonal to each other, $2 \mathrm{D}$ beam pattern of the array in elevation and azimuth direction will be mainly affected by linear and circular arrays, respectively. Therefore, the $2 \mathrm{D}$ beamforming process can be realized by two individual $1 \mathrm{D}$ pattern syntheses of the circular and linear arrays successively. Through this process, the new method can greatly decrease the amount of computation of $2 \mathrm{D}$ beamforming for cylindrical arrays.

\section{Problem Formulation}

Consider a cylindrical array consisting of $M \times N$ elements; the sketch map can be seen in Figure 1. $M$ and $N$ are the number of elements contained in each line and circle. $O(x, y, z)$ is the global Cartesian coordinate system, while $O\left(x^{\prime}, y^{\prime}, z^{\prime}\right)$ is the local system, and $a_{i}$ is the central angle of the $i$ th element. The far-field beam pattern in the generic direction $(\theta, \varphi)$ can be written as

$$
E(\theta, \varphi)=\sum_{i=1}^{M N} w_{i} g_{i}\left(\theta_{i}^{\prime}, \varphi_{i}^{\prime}\right) \exp \left\{j k \mathbf{V}_{i}^{T} \mathbf{e}(\theta, \varphi)\right\},
$$

in which $\theta$ and $\varphi$ are the azimuth and elevation, $w_{i}$ is the complex excitation voltage of the $i$ th element, $k=2 \pi / \lambda$ is the phase constant, and $g_{i}\left(\theta_{i}^{\prime}, \varphi_{i}^{\prime}\right)$ is the antenna gain of the $i$ th element with its local direction $\left(\theta_{i}^{\prime}, \varphi_{i}^{\prime}\right)$ associated with the global direction $(\theta, \varphi) . \mathbf{V}_{i}=\left[x_{i}, y_{i}, z_{i}\right]^{T}$ is the position vector of the $i$ th element, while $\mathbf{e}(\theta, \varphi)=[\cos \theta \cos \varphi, \sin \theta \cos \varphi, \sin \varphi]^{T}$ is the unit vector in the direction $(\theta, \varphi)$.

Since the array is conformal to a curved surface, the antenna elements generally direct their radiation beams toward different directions. Therefore, the transformation

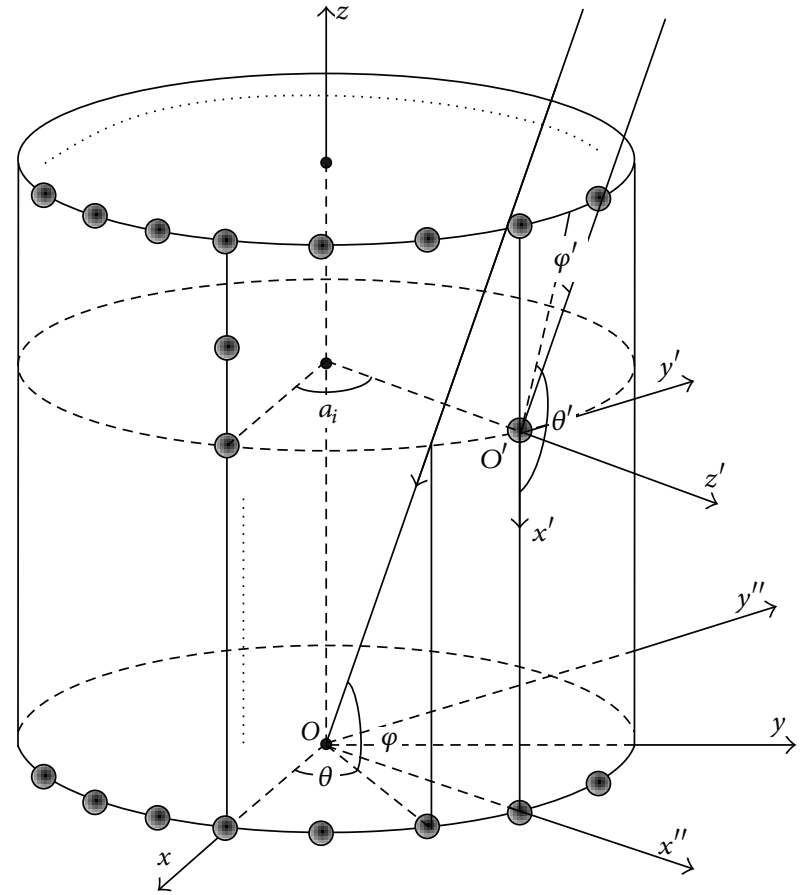

FIgURE 1: Geometry configuration and array coordinate system of cylindrical array.

between the global coordinate system and the element local coordinate system needs to be carried out to calculate the contribution of each element to the whole conformal array radiation, which is shown as follows:

$$
(\theta, \varphi) \Longleftrightarrow(x, y, z) \Longleftrightarrow\left(x^{\prime}, y^{\prime}, z^{\prime}\right) \Longleftrightarrow\left(\theta^{\prime}, \varphi^{\prime}\right)
$$

The Euler rotation matrix is a very useful tool for this spatial rotation transformation [18]. The corresponding rotation matrix can be written as

$$
\begin{aligned}
R(D, E, F) & =R_{1} R_{2} R_{3} \\
= & {\left[\begin{array}{ccc}
\cos F & \sin F & 0 \\
-\sin F & \cos F & 0 \\
0 & 0 & 1
\end{array}\right]\left[\begin{array}{ccc}
\cos E & 0 & -\sin E \\
0 & 1 & 0 \\
\sin E & 0 & \cos E
\end{array}\right] } \\
& \times\left[\begin{array}{ccc}
\cos D & \sin D & 0 \\
-\sin D & \cos D & 0 \\
0 & 0 & 1
\end{array}\right],
\end{aligned}
$$

where $[D, E, F]$ are three Euler rotation angles of the axes $O z$, $O y^{\prime \prime}$, and $O^{\prime} z^{\prime}$, respectively. And then, the spatial rotation transformation can finally be written as

$$
\left[\begin{array}{c}
\cos \theta^{\prime} \cos \varphi^{\prime} \\
\sin \theta^{\prime} \cos \varphi^{\prime} \\
\sin \varphi^{\prime}
\end{array}\right]=R(D, E, F)\left[\begin{array}{c}
\cos \theta \cos \varphi \\
\sin \theta \cos \varphi \\
\sin \varphi
\end{array}\right] .
$$


For a cylindrical array, the maximal radiation of antenna is along the normal direction at most of the time; the coordinate transformation process can be expressed as

$$
O(x, y, z) \Longrightarrow O\left(x^{\prime \prime}, y^{\prime \prime}, z\right) \Longrightarrow O\left(x^{\prime}, y^{\prime}, z^{\prime}\right)
$$

Without considering the coordinate shift, the rotation angles in (3) are $D=a_{i}, E=\pi / 2$, and $F=0$ in this case, where $a_{i}$ is the corresponding central angle of the $i$ th element.

\section{2D Beamforming for Cylindrical Arrays}

In a planar array, the array factor and the element pattern are separable, but this condition does not hold for the case of a general conformal array. Consider the structure characteristic of cylindrical array; it consists of a series of identical circular arrays and the whole array can be seen as an equivalent linear array whose elements are these identical circular subarrays. Therefore, the array beam pattern can still be obtained by the principle of pattern multiplication. And then, the $2 \mathrm{D}$ beam pattern of cylindrical array $E(\theta, \varphi)$ can be expressed by the following formula:

$$
E(\theta, \varphi)=E_{1}(\theta, \varphi) E_{2}(\theta, \varphi),
$$

where $E_{1}(\theta, \varphi)$ is the array factor of the linear array and $E_{2}(\theta, \varphi)$ is the beam pattern of the circular subarray which is used as the element pattern here. Assume that the complex excitation vector of linear array and circular array are $\mathbf{w}_{1}=\left[w_{11}, w_{12}, \ldots, w_{1 M}\right]^{T}$ and $\mathbf{w}_{2}=\left[w_{21}, w_{22}, \ldots, w_{2 N}\right]^{T}$, respectively. Then, the beam pattern $E_{1}(\theta, \varphi)$ and $E_{2}(\theta, \varphi)$ can be written as

$$
\begin{gathered}
E_{1}(\theta, \varphi)=\mathbf{w}_{1}^{T} \mathbf{a}_{1}(\theta, \varphi), \\
E_{2}(\theta, \varphi)=\mathbf{w}_{2}^{T}\left[\operatorname{diag}\{\mathbf{g}(\theta, \varphi)\} \mathbf{a}_{2}(\theta, \varphi)\right],
\end{gathered}
$$

where $\mathbf{a}_{1}(\theta, \varphi)$ and $\mathbf{a}_{2}(\theta, \varphi)$ are the ideal steering vector of the linear array and the circular array and $\mathbf{g}(\theta, \varphi)=$ $\left[g_{1}\left(\theta_{1}^{\prime}, \varphi_{1}^{\prime}\right), \ldots, g_{N}\left(\theta_{N}^{\prime}, \varphi_{N}^{\prime}\right)\right]^{T}$. The symbol $\operatorname{diag}\{\mathbf{g}\}$ represents a diagonal matrix with diagonal entries $g_{1}, \ldots, g_{N}$. Define the equivalent steering vector $\mathbf{a}_{2}^{\prime}(\theta, \varphi)=\operatorname{diag}\{\mathbf{g}(\theta, \varphi)\} \mathbf{a}_{2}(\theta, \varphi)$, and from (6), (7), and (8), beam pattern in (1) can be rewritten as follows:

$$
\begin{aligned}
E(\theta, \varphi) & =\left(\mathbf{w}_{1}^{T} \mathbf{a}_{1}(\theta, \varphi)\right)\left(\mathbf{w}_{2}^{T} \mathbf{a}_{2}^{\prime}(\theta, \varphi)\right) \\
& =\left(\mathbf{w}_{1}^{T} \otimes \mathbf{w}_{2}^{T}\right)\left(\mathbf{a}_{1}(\theta, \varphi) \otimes \mathbf{a}_{2}^{\prime}(\theta, \varphi)\right) \\
& =\mathbf{w}^{T} \mathbf{a}(\theta, \varphi),
\end{aligned}
$$

in which $\mathbf{a}(\theta, \varphi)=\mathbf{a}_{1}(\theta, \varphi) \otimes \mathbf{a}_{2}^{\prime}(\theta, \varphi)$ is the steering vector of the whole cylindrical array and its weight vector can be obtained as follows:

$$
\mathbf{w}=\mathbf{w}_{1} \otimes \mathbf{w}_{2},
$$

where " $\otimes$ " denotes the Kronecker product.

Since the array factor of linear array is identical in azimuth, it can be simplified as $E_{1}(\varphi)$. Moreover, since the linear array and circular array are orthogonal to each other, the beam pattern in the azimuth direction is mainly determined by the circular subarrays. Therefore, the $2 \mathrm{D}$ beam pattern can be decomposed into two $1 \mathrm{D}$ beamforming processes, one in the azimuth direction and one in the elevation direction. In order to achieve the desired 2D beam pattern $E_{d}(\theta, \varphi)$, we first carry out beamforming for circular subarray to achieve the given SLL in azimuth and then obtain the weight vector $\mathbf{w}_{2}$ which can satisfy the following equation:

$$
\mathbf{w}_{2}^{T} \mathbf{a}_{2}^{\prime}\left(\theta, \varphi=\varphi_{d}\right)=E_{2}\left(\theta, \varphi=\varphi_{d}\right)=E_{d}\left(\theta, \varphi=\varphi_{d}\right) .
$$

And then, $E_{2}\left(\theta=\theta_{d}, \varphi\right)$ is used as the element pattern in the beamforming for linear array in the direction of elevation to obtain the weight vector $\mathbf{w}_{1}$ which can satisfy the following equation:

$$
\begin{aligned}
\mathbf{w}_{1}^{T} \mathbf{a}_{1}(\varphi) E_{2}\left(\theta=\theta_{d}, \varphi\right) & =E_{1}(\varphi) E_{2}\left(\theta=\theta_{d}, \varphi\right) \\
& =E_{d}\left(\theta=\theta_{d}, \varphi\right) .
\end{aligned}
$$

The weight vector for the $1 \mathrm{D}$ pattern synthesis can be obtained based on an adaptive array method as follows [7]:

$$
\mathbf{w}=\mu \mathbf{R}_{i+n}^{-1} \mathbf{a}^{*}\left(\phi_{d}\right)=\mu\left(\sigma_{n}^{2} \mathbf{I}+\sum_{j=1}^{J} \sigma_{j}^{2} \mathbf{a}_{j} \mathbf{a}_{j}^{H}\right)^{-1} \mathbf{a}^{*}\left(\phi_{d}\right),
$$

where $\phi_{d}$ is the look direction, while $\sigma_{n}^{2}$ and $\sigma_{j}^{2}, j=1,2, \ldots, J$, are the power of noise and artificial interference signals, respectively. Through the following iteration, the optimal weight vector can be obtained to approach the desired beam pattern:

$$
\sigma_{j}^{2}(n+1)= \begin{cases}0 & \phi_{j} \text { in main lobe region } \\ \max \left\{0, \Gamma_{j}(n)\right\} & \phi_{j} \text { in side lobe region, }\end{cases}
$$

where $n$ denotes the $n$th iteration, $\Gamma_{j}(n)=\sigma_{j}^{2}(n)+\alpha\left[E_{n}\left(\phi_{j}\right)-\right.$ $\left.E_{d}\left(\phi_{j}\right)\right]$, and $E_{d}(\phi)$ is the desired beam pattern.

Since the radiation of array element is not isotropic, some elements may have little contribution to the look direction. And consequently we ignore these elements in the beamforming process to further decrease the computational complexity. In most cases, elements radiate outwards around the normal direction, so we only use the elements in an area around the look direction, and the subarray becomes a circular arc array as shown in Figure 2. The solid ones represent the used elements and the circle ones represent the ignored elements.

From the above, the proposed algorithm can be summarized as follows.

(1) Consider a cylindrical array consisting of $M \times N$ identical elements with their identical pattern $g(\theta, \varphi)$. Select the contributive elements as shown in Figure 2, and carry out the beamforming process for the circular arc array in azimuth with the fixed elevation $\varphi=\varphi_{d}$. Through the iteration of (13) and (14), the weight vector $\mathbf{w}_{2}$ of circular subarray and its $1 \mathrm{D}$ beam pattern $E_{2}\left(\theta, \varphi_{d}\right)$ can be obtained. 


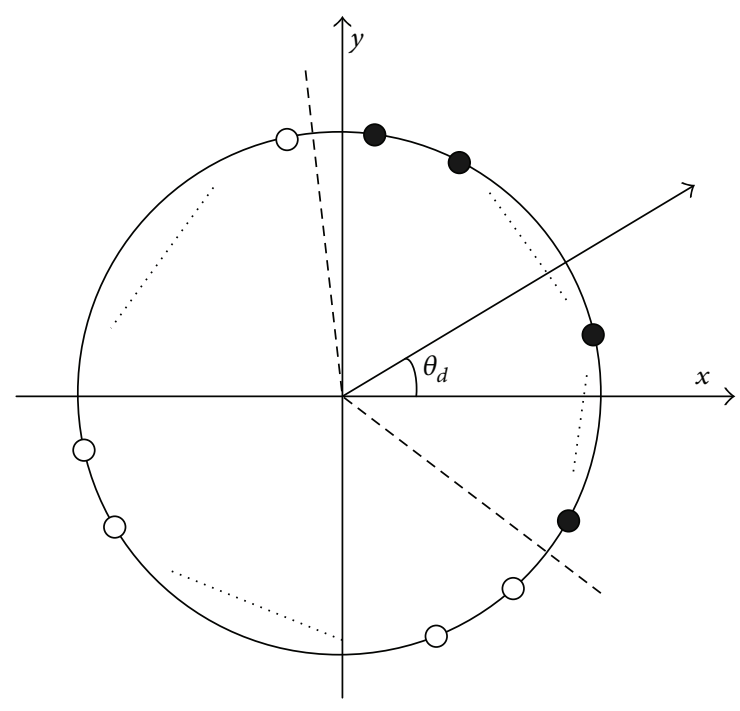

FIGURE 2: Elements used in the beamforming for circular array.

(2) Calculate the beam pattern $E_{2}\left(\theta_{d}, \varphi\right)$ of circular subarray by (8). Use it as the element pattern in the M-element linear array and similarly carry out the beamforming process in elevation with the fixed azimuth $\theta=\theta_{d}$.

(3) After getting $\mathbf{w}_{2}$ and $\mathbf{w}_{1}$ for the circular and linear arrays, respectively, the weight vector $\mathbf{w}$ of the cylindrical array can be obtained by (10), and then the $2 \mathrm{D}$ beam pattern $E(\theta, \varphi)$ can be obtained by (9) as well.

From the above, we can see that the practically used array is a part of a complete cylindrical array. Actually, the proposed method can be applied to any conformal array with similar structure that it can be seen as an equivalent linear array whose elements are identical subarrays.

\section{Results and Discussions}

In this section, simulations are provided to illustrate the effectiveness of the proposed method. Consider a uniform cylindrical array with $M=8$ and $N=25$ as shown in Figure 1. The space between neighboring elements is half wavelength. Each element is assumed to have the same pattern function $g\left(\theta_{i}, \varphi_{i}\right)=\sin \varphi_{i}, \theta_{i} \in[0,2 \pi]$, and $\varphi_{i} \in$ $[0, \pi / 2]$ in their respective local coordinate system. Since the attenuation of the pattern is more than $10 \mathrm{~dB}$ at $\varphi_{i}=15^{\circ}$ in the local coordinate system and this angle is $\theta=75^{\circ}$ in the global coordinate system, the contributive element we choose to carry out the uniform circular array beamforming is in the area $\theta \in\left[\theta_{d}-75^{\circ}, \theta_{d}+75^{\circ}\right]$ and others are ignored with this element pattern. Without loss of generality, we assume that the look direction is $\left(\theta_{d}, \varphi_{d}\right)=\left(0^{\circ}, 0^{\circ}\right)$. Then, the number of actually used elements in the uniform circular array beamforming is 11 in following simulations. The desired beam pattern is defined by the main lobe beamwidth between first nulls $\left(50^{\circ}\right)$ and side lobe level $(-40 \mathrm{~dB})$. The interested angle area is $\left[-90^{\circ}, 90^{\circ}\right] \times\left[-90^{\circ}, 90^{\circ}\right]$ with its scan step $1^{\circ}$.

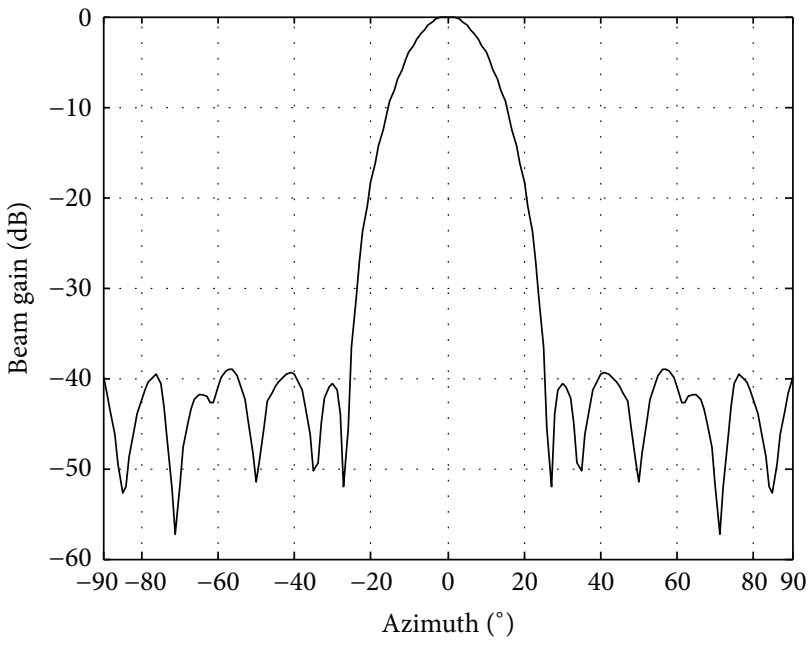

FIGURE 3: $1 \mathrm{D}$ beam pattern in the azimuth direction, with $\varphi=0^{\circ}$.

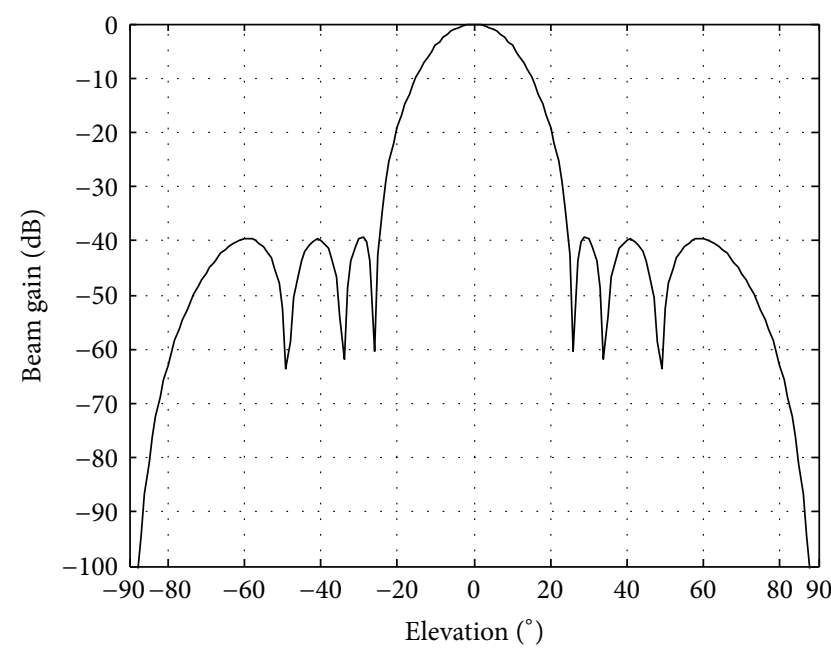

Figure 4: 1D beam pattern in the elevation direction, with $\theta=0^{\circ}$.

First, we carry out beamforming for the 11-element arc array. The artificial interferences are set with an interval of $3^{\circ}$ and the same below. After 100 iterations, the weight vector $\mathbf{w}_{2}$ is obtained and its beam pattern $E_{2}\left(\theta, \varphi=0^{\circ}\right)$ is shown in Figure 3. Consequently, $E_{2}\left(\theta=0^{\circ}, \varphi\right)$ can be obtained and is used as the element pattern for the following 8-element uniform linear array beamforming in the elevation direction. The process is similar to the above and iteration number is also set to be 100 . Figure 4 shows the final 1D beam pattern in the elevation direction.

After getting the weight vectors $\mathbf{w}_{1}$ and $\mathbf{w}_{2}$, the whole weight vector $\mathbf{w}$ of the practically used conformal array can be calculated and so is the 2D beam pattern as shown in Figure 5. Figures 6 and 7 show its projections in azimuth and elevation directions, respectively. Obviously, they coincide with the 1D beam pattern we obtained above, which well supports the analyses above. It can be seen from the figures that the finally obtained beam pattern can successfully achieve the prescribed beam pattern response. 


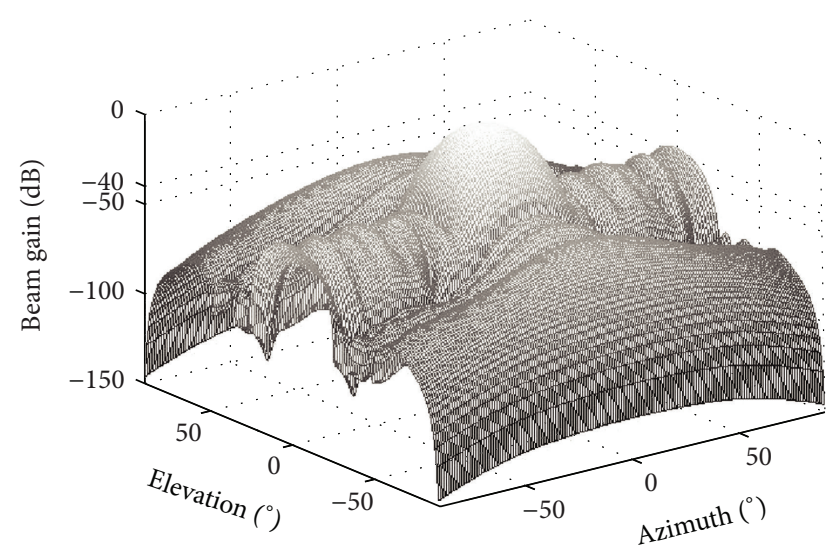

FIGURE 5: 2D beam pattern for the cylindrical array.

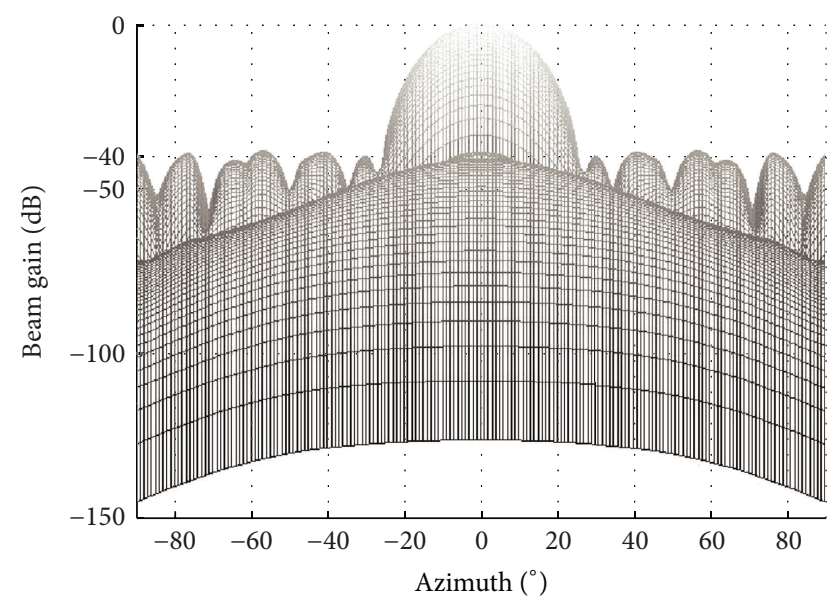

FIGURE 6: Beam pattern projection in the azimuth direction.

The method in [9] is also performed for the 2D beamforming for cylindrical array in the same condition. With the same interval, there will be $61 \times 61=3721$ artificial interferences. Result can be seen in Figure 8 which is similar to the above. However, the amount of computation is more than that of the proposed method. Figure 9 shows the normalized weights obtained by the two methods, where method 1 denotes the proposed method and method 2 denotes the method in [9].

In the pattern synthesis, the calculation mainly focuses on the spatial rotation transformation, the update of the power of artificial interferences, and matrix inversion. In our method, the whole process is mainly composed of two 1D beamforming processes. There are 61 interferences for the beamforming of each of azimuth and elevation. Assume that the number of iterations is L. Then, to get the final optimal weight vector $\mathbf{w}$, we need to calculate rotation transformation $11 \times 61$ times, matrix inversion $2 \times \mathrm{L}$ times and interference power $2 \times 61 \times \mathrm{L}$ times. Moreover, the size of matrix inversion is small $(11 \times 11$ and $8 \times 8)$. Meanwhile, if the method in [9] is directly applied to the $2 \mathrm{D}$ pattern synthesis of this cylindrical array, then we need to calculate rotation transformation $88 \times 3721$ times, matrix inversion $\mathrm{L}$ times

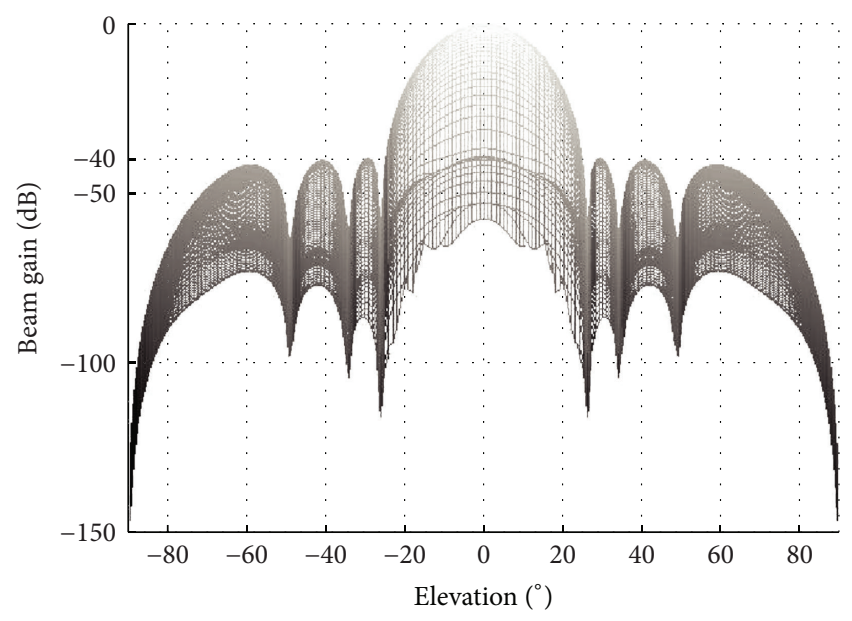

FIGURE 7: Beam pattern projection in the elevation direction.

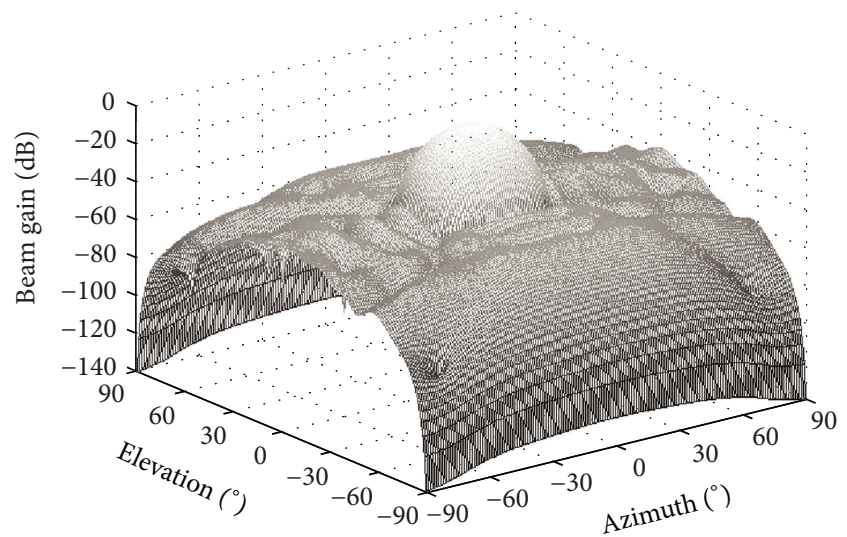

FiguRE 8: 2D beam pattern for the cylindrical array.

TABLE 1: CPU time for different array sizes.

\begin{tabular}{lcc}
\hline Array size $(M \times N)$ & $T_{1}(\mathrm{~s})$ & $T_{2}(\mathrm{~s})$ \\
\hline $8 \times 25$ & 0.671006 & 268.723427 \\
$12 \times 36$ & 0.731100 & 562.419302 \\
$20 \times 50$ & 1.116190 & 2756.073839 \\
\hline
\end{tabular}

and interference power $3721 \times \mathrm{L}$ times. The size of matrix inversion is $88 \times 88$. The number of artificial interferences and size of matrix are the main reason for the large amount of calculation. Our method can significantly reduce both of them and hence reduce the amount of calculation. Moreover, with the increase of array element number, the amount of computation will be rapidly increasing for those direct $2 \mathrm{D}$ beamforming algorithms, while our method will not. More scenarios are simulated to verify the proposed algorithm. With the Intel (R) Core (TM) i5 CPU 2.4 GHz and 4 GB RAM, the elapsed time of getting the optimal weight vector for both the proposed method and the method in [9] is shown in Table 1.

$T_{1}$ and $T_{2}$ in Table 1 are the elapsed time for the proposed method and the method in [9], respectively. Although 


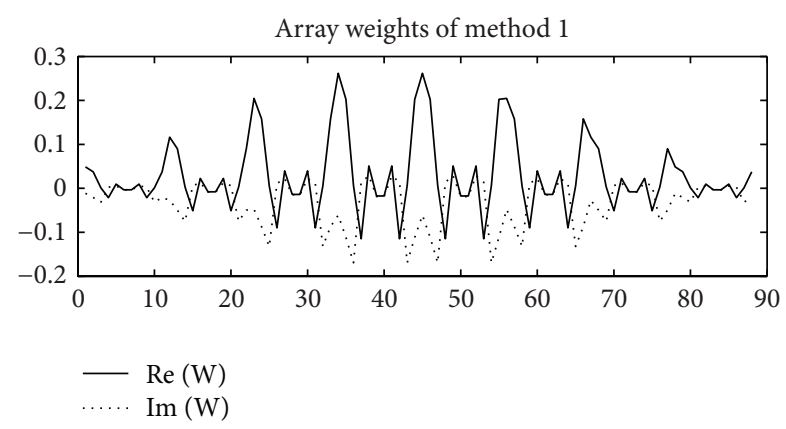

(a)

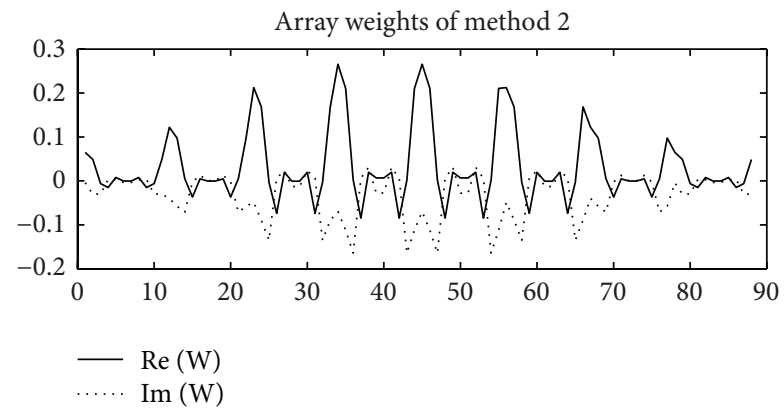

(b)

FIGURE 9: Normalized weights of the cylindrical array.

the elapsed time may be affected by the computing environment and some parameters of the conformal array, there is no doubt that our method has greatly reduced the amount of calculation without performance degradation.

\section{Conclusion}

In this paper, an efficient $2 \mathrm{D}$ beamforming method is proposed for cylindrical arrays. According to the geometry characteristics, a cylindrical array can be seen as an equivalent linear array composed of identical circular subarrays, so that the principle of pattern multiplication can be applied and $2 \mathrm{D}$ pattern synthesis process can be realized by two $1 \mathrm{D}$ pattern syntheses based on the linear and circular arrays, respectively. This method avoids the complicated calculation of 2D beam pattern and can significantly reduce the amount of computation. Furthermore, the proposed method can be applied to any similar conformal arrays which can be seen as a linear array whose elements are identical subarrays. Effectiveness of the algorithm has been illustrated by the above simulations.

\section{Acknowledgments}

This work is supported by China Postdoctoral Science Foundation Grant no. 20100480680 and the Natural Science Foundation of Anhui Province Grant no. 1208085QF105.

\section{References}

[1] L. Josefsson and P. Persson, Conformal Array Antenna Theory and Design, IEEE Press, Piscataway, NJ, USA, 2006.
[2] O. M. Bucci, G. Franceschetti, G. Mazzarella, and G. Panariello, "Intersection approach to array pattern synthesis," IEE Proceedings $H$, vol. 137, no. 6, pp. 349-357, 1990.

[3] H. Steyskal, "Pattern synthesis for a conformal wing array," IEEE Aerospace Conference Proceedings, vol. 2, pp. 819-824, 2002.

[4] C.-Y. Tseng and L. J. Griffiths, "A simple algorithm to achieve desired patterns for arbitrary arrays," IEEE Transactions on Signal Processing, vol. 40, no. 11, pp. 2737-2746, 1992.

[5] L. I. Vaskelainen, "Iterative least-squares synthesis methods for conformai array antennas with optimized polarization and frequency properties," IEEE Transactions on Antennas and Propagation, vol. 45, no. 7, pp. 1179-1185, 1997.

[6] L. I. Vaskelainen, "Constrained least-squares optimization in conformal array antenna synthesis," IEEE Transactions on Antennas and Propagation, vol. 55, no. 3, pp. 859-867, 2007.

[7] C. A. Olen and R. T. Compton Jr., "A numerical pattern synthesis algorithm for arrays," IEEE Transactions on Antennas and Propagation, vol. 38, no. 10, pp. 1666-1676, 1990.

[8] P. Y. Zhou and M. A. Ingram, "Pattern synthesis for arbitrary arrays using an adaptive array method," IEEE Transactions on Antennas and Propagation, vol. 47, no. 5, pp. 862-869, 1999.

[9] Q. Guo, G. Liao, Y. Wu, and J. Li, "Pattern synthesis method for arbitrary arrays based on LCMV criterion," Electronics Letters, vol. 39, no. 23, pp. 1628-1630, 2003.

[10] C. Dohmen, J. W. Odendaal, and J. Joubert, "Synthesis of conformal arrays with optimized polarization," IEEE Transactions on Antennas and Propagation, vol. 55, no. 10, pp. 2922-2925, 2007.

[11] L. Zou, J. Lasenby, and Z. He, "Beamforming with distortionless co-polarisation for conformal arrays based on geometric algebra," IET Radar, Sonar and Navigation, vol. 5, no. 8, pp. 842-853, 2011.

[12] T. Su and H. Ling, "Array beamforming in the presence of a mounting tower using genetic algorithms," IEEE Transactions on Antennas and Propagation, vol. 53, no. 6, pp. 2011-2019, 2005.

[13] J. O. Yang, Q. R. Yuan, F. Yang, H. J. Zhou, Z. P. Nie, and Z. Q. Zhao, "Synthesis of conformal phased array with improved NSGA-II algorithm," IEEE Transactions on Antennas and Propagation, vol. 57, no. 12, pp. 4006-4009, 2009.

[14] J. A. Ferreira and F. Ares, "Pattern synthesis of conformai arrays by the simulated annealing technique," Electronics Letters, vol. 33, no. 14, pp. 1187-1189, 1997.

[15] Z. B. Lu, A. Zhang, and X. Y. Hou, "Pattern synthesis of cylindrical conformal array by the modified particle swarm optimization algorithm," Progress in Electromagnetics Research, vol. 79, pp. 415-426, 2008.

[16] M. Comisso and R. Vescovo, "Fast iterative method of power synthesis for antenna arrays," IEEE Transactions on Antennas and Propagation, vol. 57, no. 7, pp. 1952-1962, 2009.

[17] Z. Lin and H. Zishu, "A beamforming method for cylindrical array based on synthetic pattern of subarray," in Proceedings of the 2nd International Conference on Image Analysis and Signal Processing (IASP '10), pp. 667-670, April 2010.

[18] T. Milligan, "More applications of Euler rotation angles," IEEE Antennas and Propagation Magazine, vol. 41, no. 4, pp. 78-83, 1999. 

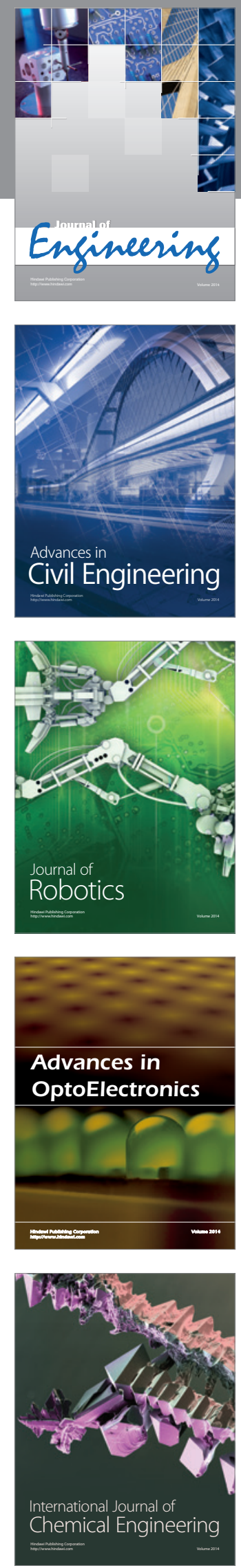

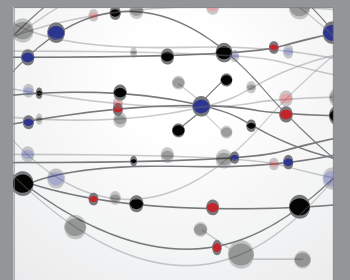

The Scientific World Journal
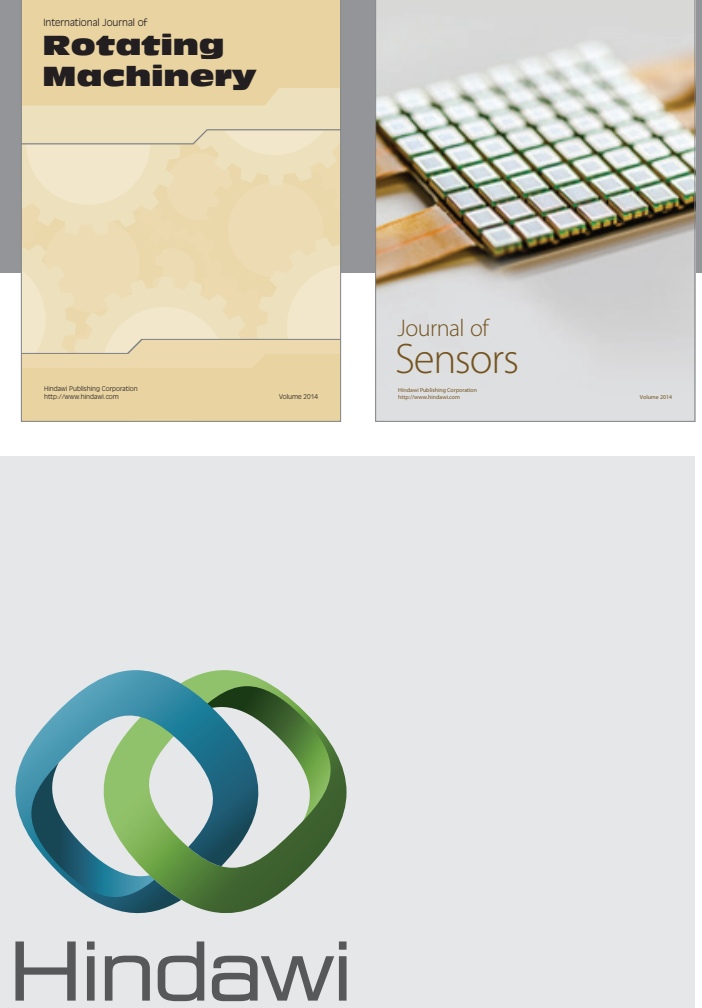

Submit your manuscripts at http://www.hindawi.com
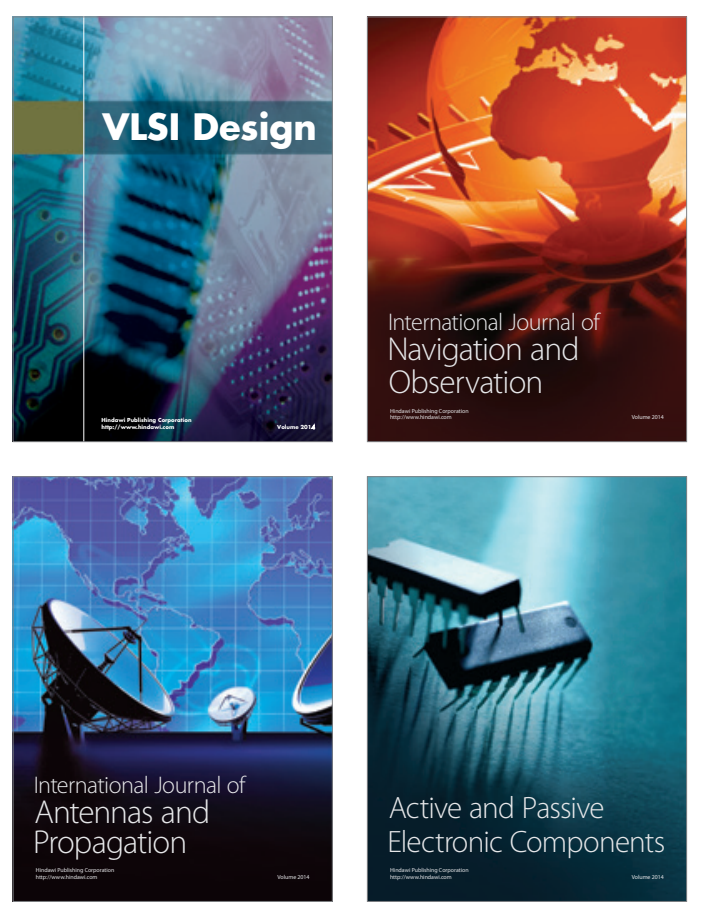
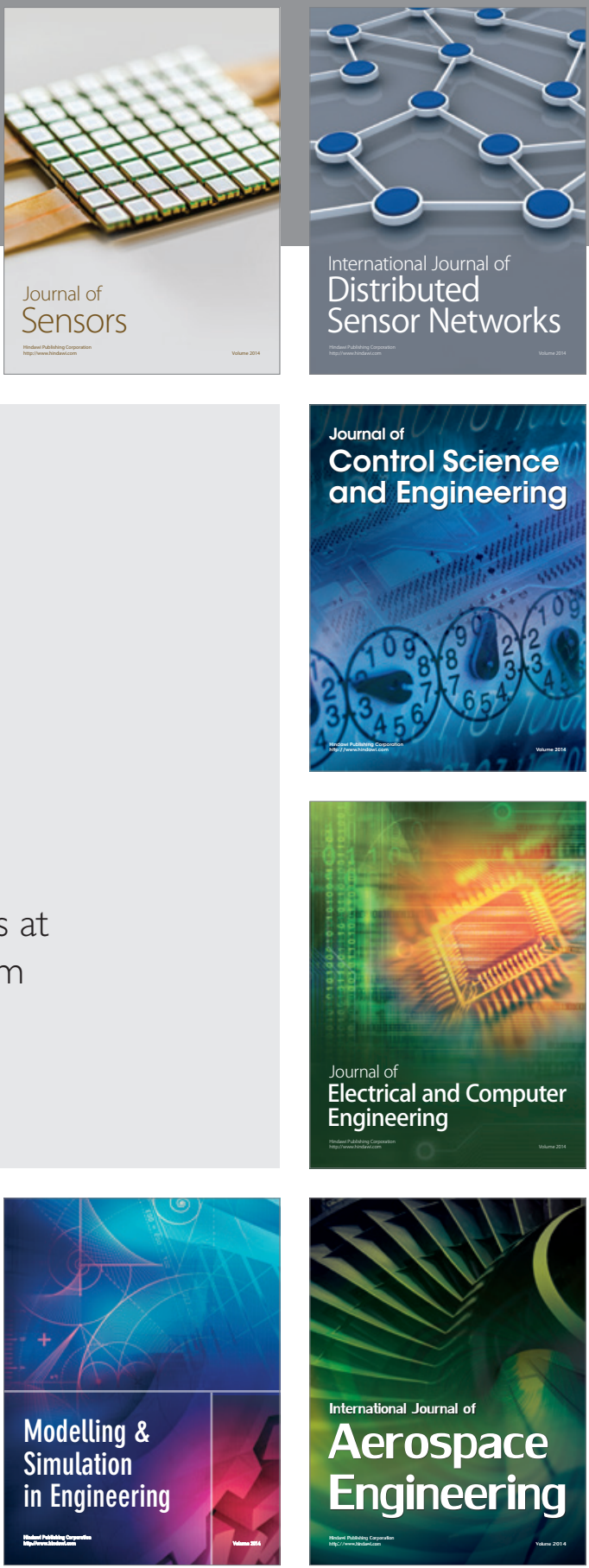

Journal of

Control Science

and Engineering
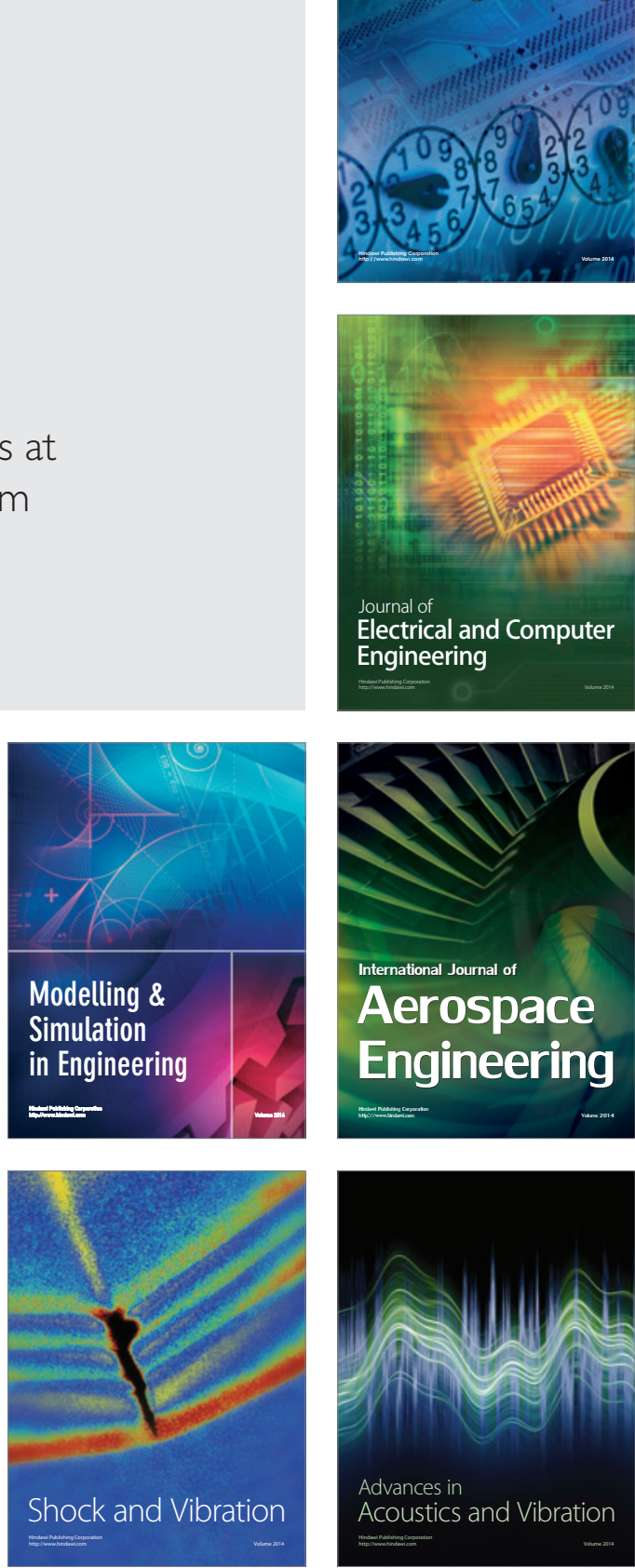